\title{
Epi-Lipschitzian reachable sets of differential inclusions
}

\author{
Thomas Lorenz \\ Interdisciplinary Center for Scientific Computing, University of Heidelberg, \\ Im Neuenheimer Feld 294, 69120 Heidelberg, Germany ${ }^{1}$
}

\begin{abstract}
The reachable sets of a differential inclusion have nonsmooth topological boundaries in general. The main result of this paper is that under the well-known assumptions of Filippov's existence theorem (about solutions of differential inclusions), every epi-Lipschitzian initial compact set $K \subset \mathbb{R}^{N}$ preserves this regularity for a short time, i.e. $\vartheta_{F}(t, K)$ is also epi-Lipschitzian for all small $t>0$.

The proof is based on Rockafellar's geometric characterization of epi-Lipschitzian sets and uses a new result about the "inner semicontinuity" of Clarke tangent cone $(t, y) \mapsto T_{\vartheta_{F}(t, K)}^{C}(y) \subset \mathbb{R}^{N}$ with respect to both arguments.
\end{abstract}

Key words: Differential inclusion, reachable set (alias attainable set), epi-Lipschitzian sets, Clarke tangent cone, lower limit (in the sense of Painlevé-Kuratowski)

\section{Introduction}

Differential inclusions represent an important analytical tool for describing dynamic systems with aspects of uncertainties. Historically speaking, so-called control systems $x^{\prime}(t)=$ $f(x(t), u(t), t)$ extended the classical field of ordinary differential equations by means of a (possibly) time-dependent parameter $u(t) \in U$ (but under hardly any regularity assumptions about $u(\cdot)) \cdot u(\cdot)$ is usually called control function. Dispensing now with its exact value, we find a differential inclusion $x^{\prime}(t) \in F(x(t), t)$ with a multivalued map $F: \mathbb{R}^{N} \times[0, T] \leadsto \mathbb{R}^{N}$ on its right-hand side. (In more popular terms, such a multivalued map $F$ is a function on $\mathbb{R}^{N} \times[0, T]$ whose values are subsets of $\mathbb{R}^{N}$ - not necessarily consisting of just one element.)

Obviously, in each initial point $x \in \mathbb{R}^{N}$, more than only one solution $x(\cdot) \in W^{1,1}\left([0, t], \mathbb{R}^{N}\right)$ of $x^{\prime}(\cdot) \in F(x(\cdot), \cdot)$ a.e. might start. More generally speaking, the so-called reachable set

\footnotetext{
Email address: thomas.lorenz@iwr.uni-heidelberg.de (Thomas Lorenz).

1 The project was partially funded by SFB 359 "Reactive flow, diffusion and transport" of the German Research Foundation (DFG). The author thanks the referee for his very detailed comments and, he is grateful to Irina Surovtsova and Daniel Andrej for fruitful complementary discussions.
} 
$\vartheta_{F}(t, K) \subset \mathbb{R}^{N}$ (alias attainable set) of a subset $K \subset \mathbb{R}^{N}$ at time $t \in[0, T]$ is defined as set of all points that can be attained at time $t$ by an absolutely continuous solution starting in $K$, i.e.

$$
\begin{aligned}
\vartheta_{F}(t, K):=\left\{x(t) \in \mathbb{R}^{N} \mid \exists x(\cdot) \in W^{1,1}\left([0, t], \mathbb{R}^{N}\right): x(0) \in K,\right. \\
\left.x^{\prime}(\cdot) \in F(x(\cdot), \cdot) \text { almost everywhere in }[0, t]\right\} .
\end{aligned}
$$

This paper focuses on the regularity of the topological boundary of $\vartheta_{F}(t, K)$ after a short time $t>0$.

For Lipschitz continuous multivalued maps $F$ with nonempty compact values, the reachable set $\vartheta_{F}(t, K)$ of any compact initial set $K \subset \mathbb{R}^{N}$ is known to be also compact at any time $t \in[0, \infty$ [ - due to Filippov's Theorem and Gronwall's Lemma (see e.g. [2, § 3.7.1]). Smooth boundaries, however, need not stay smooth in the long run. The regularity of $\partial \vartheta_{F}(t, K) \subset \mathbb{R}^{N}$ has usually been investigated under very strong assumptions about the structure of $F$ (e.g. affine-linear maps) and about the initial set $K \subset \mathbb{R}^{N}$ (such as convexity or smooth boundary). Bressan's paper [8] exemplifies early results about reachable sets (of a single point) with $C^{1}$ boundary in affine-linear control systems with constant smooth control sets. Recently, Cannarsa and Frankowska published sufficient conditions on control systems for the interior sphere property of their reachable sets [9]. As a consequence, they obtain even sufficient conditions on control system and initial convex set such that its reachable set has $C^{1,1}$ boundary at all small positive times [9, Corollary 3.12]. Independently from them, the author specified other sufficient conditions for preserving a similar property (called positive erosion) of the compact initial set [13], [12, Appendix A].

Now Lipschitz continuity is in the focus of interest: Assume that the initial compact set $K \subset \mathbb{R}^{N}$ has Lipschitz boundary and is locally on one side of $\partial K$. In other words, it is locally "above" the graph of a Lipschitz continuous function. This is the geometric interpretation of a so-called epi-Lipschitzian set - a term introduced and characterized by Rockafellar in 1979 [14] (see Definition 2.2 below).

In this paper, we show that the reachable sets $\vartheta_{F}(t, K) \subset \mathbb{R}^{N}$ then have to preserve this property for a (maybe) short time whenever the well-known existence theorem of Filippov (about solutions of differential inclusions) can be applied.

This result pertains to a set feature completely different from the interior sphere property in [9] and positive erosion in [13]. Indeed, roughly speaking, all types of "corner" (with positive interior and exterior angles) can be taken under consideration here. In control problems and calculus of variational, sets of constraints and graphs have sometimes been assumed as epi-Lipschitzian. So our result here comes into play when these sets are not fixed in time, but deformed according to a differential inclusion.

The proof is based on a geometric argument seizing Rockafellar's characterization of epiLipschitzian sets (see Lemma 3.1 below). So we use the Clarke tangent cone $T_{K}^{C}(\cdot)$ specified in Definition 2.1 and its properties. These tools for nonsmooth sets differ conceptually from the more "analytically oriented" description of subsets as sublevel sets of a sufficiently smooth (but single-valued) function, see e.g. $[1,16]$. In regard to reachable sets, we prove a form of "inner semicontinuity" $[15, \S 5$.B $]$ of the multivalued map $(t, x) \longmapsto T_{\vartheta_{F}(t, K)}^{C}(x)$ for every compact epi-Lipschitzian set $K \subset \mathbb{R}^{N}$, i.e.

$$
T_{K}^{C}\left(x_{0}\right)=\operatorname{Liminf}_{\substack{t \geq 0 \\ y \rightarrow x_{0} \\\left(y \in \vartheta_{F}(t, K)\right)}} T_{\vartheta_{F}(t, K)}^{C}(y)
$$


with the lower limit denoted as Liminf in the sense of Painlevé-Kuratowski $[4, \S 1.4]$ (see Proposition 2.4 below).

Finally we sketch the structure of this paper: In $\S 2$, the main theorem is formulated after introducing the required terms. The detailed proofs follow in $\S 3$.

\section{Main theorem and auxiliary results}

The main result of this paper concerns reachable sets of differential inclusions and the regularity of their topological boundaries. In a word, consider a differential inclusion $x^{\prime}(\cdot) \in F(x(\cdot), \cdot)$ whose multivalued function $F: \mathbb{R}^{N} \times[0, T] \leadsto \mathbb{R}^{N}$ satisfies the assumptions of Filippov's existence theorem. If the initial compact set $K \subset \mathbb{R}^{N}$ has a Lipschitz boundary, this property is shortly preserved for its reachable sets $\vartheta_{F}(t, K) \subset \mathbb{R}^{N}$.

Now we specify the terms required for the exact formulation of the main theorem. Following definitions of Clarke and Rockafellar,

Definition 2.1 ([4, $\S 4.1 .3],[10, \S 2.4]) \quad$ Let $K$ be a nonempty subset of a normed vector space $X$ and $x$ belong to its closure $\bar{K} \subset X$. The Clarke tangent cone $T_{K}^{C}(x)$ at $x$ is defined as

$$
T_{K}^{C}(x):=\left\{v \in X \mid \limsup _{h \downarrow 0, y \underset{K}{\longrightarrow} x} \frac{1}{h} \cdot \operatorname{dist}(y+h v, K)=0\right\} .
$$

Obviously, $T_{K}^{C}(x) \subset X$ is nonempty (as it contains $0 \in X$ ) and a cone, i.e. $v \in T_{K}^{C}(x)$ implies $\lambda v \in T_{K}^{C}(x)$ for any $\lambda \geq 0$. Moreover, it is always convex and closed [14],[10, $\left.\S 2.4\right]$.

Definition 2.2 ([14], $[10, \S 7.3]) \quad$ A nonempty set $K \subset \mathbb{R}^{N}$ is called epi-Lipschitzian at $x \in K$ if there exist an open neighborhood $U \subset \mathbb{R}^{N}$ of $x$, a linear map $A: \mathbb{R}^{N} \longrightarrow$ $\mathbb{R}^{N-1} \times \mathbb{R}$ and a function $\varphi: \mathbb{R}^{N-1} \longrightarrow \mathbb{R}$ satisfying

$$
K \cap U=U \cap A^{-1}(\text { epi } \varphi)
$$

with $\varphi$ being Lipschitz continuous close to the first $N-1$ real coordinates of $A(x)$ and epi $\varphi:=\{(y, \xi) \mid \xi \geq \varphi(y)\} \subset \mathbb{R}^{N-1} \times \mathbb{R}$ denoting the epigraph of $\varphi$.

$K \subset \mathbb{R}^{N}$ is called epi-Lipschitzian if $K$ is epi-Lipschitzian at each of its elements.

For applying Filippov's existence theorem about solutions of differential inclusions (Lemma 3.3 below) in a convenient way later, we introduce the property "Filippov continuous" for a multivalued map $F: \mathbb{R}^{N} \times[0, T] \leadsto \mathbb{R}^{N}$ of space and time. It reflects the gist of the feature "measurable/Lipschitz" defined in [4, Definition 9.5.1] - but in a more detailed formulation.

Definition 2.3 A multivalued map $F: \mathbb{R}^{N} \times[0, T] \leadsto \mathbb{R}^{N}$ is called Filippov continuous if it satisfies the following conditions: 
1.) the values of $F$ are nonempty closed subsets of $\mathbb{R}^{N}$,

2.) Graph $F \subset \mathbb{R}^{N} \times[0, T] \times \mathbb{R}^{N}$ belongs to $\mathcal{L}^{N} \otimes \mathcal{L}^{1} \otimes \mathcal{B}^{N}$,

3.) F has at most linear growth, i.e. $\sup _{(x, t) \in \mathbb{R}^{N} \times[0, T]} \sup _{y \in F(x, t)} \frac{|y|}{|x|+|t|+1}<\infty$.

4.) there is some $\lambda(\cdot) \in L^{1}([0, T], \mathbb{R})$ such that for almost every $t \in[0, T]$, the multivalued map $F(\cdot, t): \mathbb{R}^{N} \leadsto \mathbb{R}^{N}$ is $\lambda(t)$-Lipschitz (w.r.t. Hausdorff distance).

Here $\mathcal{L}^{N}$ consists of all Lebesgue subsets of $\mathbb{R}^{N}$ and, $\mathcal{B}^{N}$ denotes the set of all Borel subsets of $\mathbb{R}^{N}$. Condition (2.) is equivalent to the measurability of the multivalued map $F$ as shown in $[4, \S 8.1]$. Furthermore, the linear growth condition (3.) implies first that all values of $F$ are compact and second that Gronwall's Lemma provides locally uniform bounds for solutions of the corresponding nonautonomous differential inclusion.

Our contribution now is to prove an "inner semicontinuity" property of Clarke tangent cone $T_{K}^{C}(x)$ with respect to both the point $x$ and the set $K \subset \mathbb{R}^{N}$. In particular, compact sets are "deformed" along differential inclusions, i.e. for a given Filippov continuous multivalued map $F(\cdot, \cdot)$, we start with a compact initial set $K \subset \mathbb{R}^{N}$ and consider the time-dependent compact reachable sets $t \mapsto \vartheta_{F}(t, K)$. This leads to a set-valued map of two arguments, namely $(t, y) \mapsto T_{\vartheta_{F}(t, K)}^{C}(y) \subset \mathbb{R}^{N}$, always considered under the constraint $y \in \vartheta_{F}(t, K)$. We investigate a semicontinuity property for $t \searrow 0$ and $y \rightarrow x \in K$ :

Proposition 2.4 Let $F: \mathbb{R}^{N} \times[0, T] \leadsto \mathbb{R}^{N}$ be a Filippov continuous multivalued map and $K \subset \mathbb{R}^{N}$ be nonempty compact.

If the interior of the Clarke tangent cone $T_{K}^{C}\left(x_{0}\right)$ at $x_{0} \in K$ is nonempty, then

$$
T_{K}^{C}\left(x_{0}\right)=\operatorname{Liminf}_{\substack{t \geq 0 \\ y \rightarrow x_{0} \\\left(y \in \vartheta_{F}(t, K)\right)}} T_{\vartheta_{F}(t, K)}^{C}(y)
$$

with the lower limit in the sense of Painlevé-Kuratowski $[4, \S 1.4]$, i.e.

$$
\begin{aligned}
\operatorname{Liminf}_{\substack{t \rightarrow 0 \\
y \rightarrow x_{0} \\
\left(y \in \vartheta_{F}(t, K)\right)}} T_{\vartheta_{F}(t, K)}^{C}(y):=\left\{\xi \in \mathbb{R}^{N} \mid\right. & \operatorname{dist}\left(\xi, T_{\vartheta_{F}(t, K)}^{C}(y)\right) \longrightarrow 0 \\
& \text { for } \left.t \rightarrow 0, y \rightarrow x_{0} \text { with } t \geq 0, y \in \vartheta_{F}(t, K)\right\} .
\end{aligned}
$$

For any compact epi-Lipschitzian set $K \subset \mathbb{R}^{N}$, the Clarke tangent cone $T_{K}^{C}(\cdot): K \leadsto \mathbb{R}^{N}$ is already known to be inner semicontinuous (in the sense of [15, Definition 5.4]), i.e. $T_{K}^{C}\left(x_{0}\right)=\operatorname{Liminf}_{y} \underset{K}{\longrightarrow} x_{0} T_{K}^{C}(y)$. Indeed, its polar cone called Clarke normal cone has closed graph ([14, Corollary 2] and mentioned in [11, § 2]) and, [17, Lemma 4.10.6] bridges the gap to Clarke tangent cones via duality.

For taking "perturbations" of the set into consideration, $[4, \S 4.6]$ defines the so-called circatangent cone to the sequence $\left(K_{n}\right)_{n \in \mathbb{N}}$ of closed subsets (of a normed vector space) at a point $x \in \operatorname{Liminf}_{n \rightarrow \infty} K_{n}$ as

$$
\operatorname{Liminf}_{n \rightarrow \infty, K_{n} \ni x_{n} \rightarrow x, h_{n} \downarrow 0} \frac{1}{h_{n}}\left(K_{n}-x_{n}\right) .
$$

Obviously it coincides with the Clarke tangent cone $T_{K}^{C}(x)$ if $K_{n}=K$ for each $n$, but it depends on the sequence $\left(K_{n}\right)_{n \in \mathbb{N}}$ explicitly. In Prop. 2.4, however, we consider "classical" Clarke tangent cones $T_{\vartheta_{F}(t, K)}^{C}(y), t>0$, and $T_{K}^{C}(x)$ independently from each other. 
In [5], Benoist extended the concept of Lasry-Lions regularized functions to subsets of the Euclidean space by using Minkowski sum and complement operator. These examples of morphological operations use fixed closed (often convex) subsets as "structural elements" (i.e. roughly speaking, playing the role of the unit ball). In Proposition 2.4 here, the initial set $K \subset \mathbb{R}^{N}$ is deformed along a differential inclusion $x^{\prime}(\cdot) \in F(x(\cdot), \cdot)$ which can be interpreted as a morphological operation whose structural element depends on space and time, namely $F(x, t) \subset \mathbb{R}^{N}$ (see e.g. [2] for more details about morphological operations and differential inclusions). Thus, Benoist's results about normal cones (and their semicontinuity features) cover a different type of deformation.

So the main result can now be formulated precisely:

Theorem 2.5 Let $F: \mathbb{R}^{N} \times[0, T] \leadsto \mathbb{R}^{N}$ be a Filippov continuous multivalued map. Then for each epi-Lipschitzian compact set $K \subset \mathbb{R}^{N}$, there exists a positive time $t_{K} \leq T$ such that each reachable set $\vartheta_{F}(t, K) \subset \mathbb{R}^{N}$ for $t \in\left[0, t_{K}\right]$ is also epi-Lipschitzian.

\section{Proof of the main theorem}

The proof of this main theorem is based on a geometric characterization of epi-Lipschitzian sets which goes back to Rockafellar.

Lemma 3.1 (Rockafellar [14, Theorem 3], [10, Theorem 7.3.1]) A nonempty closed subset $K \subset \mathbb{R}^{N}$ is epi-Lipschitzian at $x \in K$ if and only if the interior of $T_{K}^{C}(x) \subset \mathbb{R}^{N}$ is nonempty.

Remark. In [7], Borwein and Strojwas introduce the property "compactly epi-Lipschitzian" and prove the corresponding characterization for any normed vector space (of possibly infinite dimension, see also [6] for more details).

In a word, Proposition 2.4 lays the basis for proving indirectly that whenever all Clarke tangent cones of a compact initial set $K \subset \mathbb{R}^{N}$ have nonempty interior, then the reachable sets $\vartheta_{F}(t, K) \subset \mathbb{R}^{N}(t>0)$ cannot loose this property immediately.

In the same paper [14], a geometric characterization of the interior of Clarke tangent cone $T_{K}^{C}(x)$ is provided. Its elements are also called hypertangents to $K$ at $x[10, \S \S 2.4,2.5]$.

Lemma 3.2 (Rockafellar [14, Theorem 2], [15, Theorem 6.36]) Let $K \subset \mathbb{R}^{N}$ be a closed set and $x \in K$. Then the interior of Clarke tangent cone to $K$ at $x$ satisfies

$$
\begin{aligned}
T_{K}^{C}(x)^{\circ} & =\left\{v \in \mathbb{R}^{N} \mid \exists \varepsilon>0:\left(K \cap \mathbb{B}_{\varepsilon}(x)\right)+\right] 0, \varepsilon\left[\cdot \mathbb{B}_{\varepsilon}(v) \subset K\right\} \\
& =\left\{v \in \mathbb{R}^{N} \mid \exists \varepsilon>0 \quad \forall y \in K \cap \mathbb{B}_{\varepsilon}(x), w \in \mathbb{B}_{\varepsilon}(v), \tau \in\right] 0, \varepsilon[: y+\tau \cdot w \in K\}
\end{aligned}
$$

with $\mathbb{B}_{\varepsilon}(v)$ abbreviating the closed ball $\mathbb{B}_{\varepsilon}(v):=\left\{w \in \mathbb{R}^{N}|| w-v \mid \leq \varepsilon\right\}$ and $U^{\circ}$ denoting always the interior of a set $U$. 
In regard to differential inclusions in the Euclidean space, the key tool is the following generalization of Filippov's classical theorem:

Lemma 3.3 (Generalized Theorem of Filippov [17, Theorem 2.4.3])

Let $\mathcal{O}$ be a relatively open subset of $\mathbb{R}^{N} \times[0, T]$. Take a set-valued map $G: \mathcal{O} \leadsto \mathbb{R}^{N}$, an arc $y(\cdot) \in W^{1,1}\left([0, T], \mathbb{R}^{N}\right)$, a point $\eta \in \mathbb{R}^{N}$ and $\left.\left.\delta \in\right] 0, \infty\right]$ such that

Assume that

$$
\mathcal{N}(y, \delta):=\bigcup_{0 \leq t \leq T} \mathbb{B}_{\delta}(y(t)) \times\{t\} \subset \mathcal{O}
$$

(i) $\quad G(z, t) \neq \emptyset$ is closed for every $(z, t) \in \mathcal{N}(y, \delta)$

(ii) Graph $G$ belongs to $\mathcal{L}^{N} \otimes \mathcal{L}^{1} \otimes \mathcal{B}^{N}$,

(iii) there exists $\lambda(\cdot) \in L^{1}([0, T])$ such that $G\left(z_{1}, t\right) \subset G\left(z_{2}, t\right)+\lambda(t)\left|z_{1}-z_{2}\right| \cdot \mathbb{B}_{1}$ for all $z_{1}, z_{2} \in \mathbb{B}_{\delta}(y(t))$ and almost every $t \in[0, T]$.

Suppose further $e^{\|k\|_{L^{1}}} \cdot\left(|\eta-y(0)|+\int_{0}^{T} \operatorname{dist}\left(y^{\prime}(t), G(y(t), t)\right) d t\right) \leq \delta$.

Then there exists a trajectory $x(\cdot) \in W^{1,1}\left([0, T], \mathbb{R}^{N}\right)$ of $x^{\prime}(\cdot) \in G(x(\cdot), \cdot)$ a.e. satisfying $x(0)=\eta \quad$ and for all $t \in[0, T]$

$$
|x(t)-y(t)| \leq|\eta-y(0)| e^{\int_{0}^{t} \lambda d r}+\int_{0}^{t} e^{\int_{s}^{t} \lambda d r} \operatorname{dist}\left(y^{\prime}(s), G(y(s), s)\right) d s .
$$

In comparison with [17, Theorem 2.4.3], the slightly modified formulation of hypothesis (ii) is based on the characterization of measurable set-valued maps with closed values shown in $[4, \S 8.1]$. These tools are now used for proving the following (rather technical) lemma:

Lemma 3.4 Let $F: \mathbb{R}^{N} \times[0, T] \leadsto \mathbb{R}^{N}$ be a Filippov continuous multivalued map, $K \subset \mathbb{R}^{N}$ a nonempty compact subset and $x_{0} \in K, v \in \mathbb{R}^{N}$. Assume for the sequences $\left(t_{n}\right)_{n \in \mathbb{N}}$ in $\left[0, \infty\left[\right.\right.$ and $\left(y_{n}\right)_{n \in \mathbb{N}}$ in $\mathbb{R}^{N}$ that

$$
\left\{\begin{array}{lll}
t_{n} \longrightarrow 0, & y_{n} \longrightarrow x_{0} & \text { for } n \rightarrow \infty \\
y_{n} \in \vartheta_{F}\left(t_{n}, K\right), & v \notin T_{\vartheta_{F}\left(t_{n}, K\right)}^{C}\left(y_{n}\right) & \text { for all } n \in \mathbb{N} .
\end{array}\right.
$$

Then, $v$ does not belong to the interior of $T_{K}^{C}\left(x_{0}\right)$.

Proof. Seizing Lemma 3.2 of Rockafellar, we verify that for each $\varepsilon>0$, there exist $y \in K \cap \mathbb{B}_{\varepsilon}\left(x_{0}\right), w \in \mathbb{B}_{\varepsilon}(v)$ and $\left.\tau \in\right] 0, \varepsilon[$ with $y+\tau \cdot w \notin K$.

As an immediate consequence of Definition 2.1,

$$
\begin{gathered}
T_{\vartheta_{F}\left(t_{n}, K\right)}^{C}\left(y_{n}\right)=\left\{w \in \mathbb{R}^{N} \mid \forall \varepsilon>0 \exists \delta>0: \operatorname{dist}\left(y+\tau \cdot w, \vartheta_{F}\left(t_{n}, K\right)\right) \leq \varepsilon \tau\right. \\
\text { for all } \left.\tau \in] 0, \delta], \quad y \in \mathbb{B}_{\delta}\left(y_{n}\right) \cap \vartheta_{F}\left(t_{n}, K\right)\right\} .
\end{gathered}
$$

So assuming $v \notin T_{\vartheta_{F}\left(t_{n}, K\right)}^{C}\left(y_{n}\right)$ for each $n \in \mathbb{N}$ implies the existence of a sequence $\left(\varepsilon_{n}\right)_{n \in \mathbb{N}}$ such that for each $n \in \mathbb{N}$ and $\delta>0$, there exist $\tau \in] 0, \delta]$ and $y \in \mathbb{B}_{\delta}\left(y_{n}\right) \cap \vartheta_{F}\left(t_{n}, K\right)$ satisfying $\left(y+\tau \mathbb{B}_{\varepsilon_{n}}(v)\right) \cap \vartheta_{F}\left(t_{n}, K\right)=\mathbb{B}_{\tau \varepsilon_{n}}(y+\tau v) \cap \vartheta_{F}\left(t_{n}, K\right)=\emptyset$. 
Setting $\delta:=\frac{1}{n}$ respectively, we obtain sequences $\left(\tau_{n}\right)_{n \in \mathbb{N}}, \quad\left(\widetilde{y}_{n}\right)_{n \in \mathbb{N}}$ such that $0<\tau_{n} \leq \frac{1}{n}$, $\widetilde{y}_{n} \in \mathbb{B}_{\frac{1}{n}}\left(y_{n}\right) \cap \vartheta_{F}\left(t_{n}, K\right)$ and $\left(\widetilde{y}_{n}+\tau_{n} \mathbb{B}_{\varepsilon_{n}}(v)\right) \cap \vartheta_{F}\left(t_{n}, K\right)=\emptyset$ for each index $n \in \mathbb{N}$. In particular, $\tau_{n} \longrightarrow 0$ and $\widetilde{y}_{n} \longrightarrow x_{0}$ for $n \rightarrow \infty$ due to $y_{n} \longrightarrow x_{0}$.

Now each point $\widetilde{y}_{n}$ belongs to the reachable set $\vartheta_{F}\left(t_{n}, K\right) \subset \mathbb{R}^{N}$ and thus, there is a corresponding solution $z_{n}(\cdot) \in W^{1,1}\left(\left[0, t_{n}\right], \mathbb{R}^{N}\right)$ of $z_{n}^{\prime}(\cdot) \in F\left(z_{n}(\cdot), \cdot\right)$ a.e. with $z_{n}(0) \in K$, $z_{n}\left(t_{n}\right)=\widetilde{y}_{n}$.

Then, $z_{n}(0) \longrightarrow x_{0}$ for $n \rightarrow \infty$. Indeed, we obtain $\left|z_{n}(0)-\widetilde{y}_{n}\right|=\left|z_{n}(0)-z\left(t_{n}\right)\right| \leq$ $t_{n} \cdot \sup _{t \in\left[0, t_{n}\right]} \sup \left|F\left(z_{n}(t), t\right)\right|$. The linear growth assumption about $F$ and Gronwall's Lemma ensure that all reachable sets $\vartheta_{F}(t, K) \subset \mathbb{R}^{N}(t \in[0, T])$ of the compact set $K$ are uniformly bounded. So, $\left|z_{n}(0)-\widetilde{y}_{n}\right| \leq c \cdot t_{n} \longrightarrow 0$ for $n \rightarrow \infty$ with a constant $c<\infty$ depending just on the maximal time $T<\infty$, the linear growth rate of $F$ and $\max _{x \in K}|x|$.

The generalized form of Filippov's Theorem (quoted in Lemma 3.3) is now used for "tracing" each point $\widetilde{y}_{n}+\tau_{n} \cdot v \notin \vartheta_{F}\left(t_{n}, K\right), n \in \mathbb{N}$, back in time to $t=0$. It will provide an initial point $z_{n}(0)+\tau_{n}\left(v+w_{n}\right) \notin K$ with $\max \left\{\left|w_{n}\right|, \tau_{n}\right\} \longrightarrow 0$ for $n \rightarrow \infty$ and thus lays the wanted basis for completing this proof.

Indeed, considering $z_{n}(\cdot)$ in reverse time direction, $z_{n}\left(t_{n}-\cdot\right):\left[0, t_{n}\right] \longrightarrow \mathbb{R}^{N}$ solves the differential inclusion $\xi^{\prime}(\cdot) \in-F\left(\xi(\cdot), t_{n}-\cdot\right)$ a.e. and starts in $\widetilde{y}_{n}$. The "translated" curve $\left[0, t_{n}\right] \longrightarrow \mathbb{R}^{N}, t \longmapsto z_{n}\left(t_{n}-t\right)+\tau_{n} v$, however, looses this solution property, but starts in the desired point $\widetilde{y}_{n}+\tau_{n} \cdot v \notin \vartheta_{F}\left(t_{n}, K\right)$. Now Lemma 3.3 ensures the existence of an absolutely continuous solution $\widetilde{z}_{n}(\cdot):\left[0, t_{n}\right] \longrightarrow \mathbb{R}^{N}$ of

$$
\widetilde{z}_{n}^{\prime}(\cdot) \in-F\left(\widetilde{z}_{n}(\cdot), t_{n}-\cdot\right) \text { a.e. in }\left[0, t_{n}\right], \quad \widetilde{z}_{n}(0)=\widetilde{y}_{n}+\tau_{n} \cdot v \notin \vartheta_{F}\left(t_{n}, K\right)
$$

satisfying additionally

$$
\left|\widetilde{z}_{n}(t)-\left(z_{n}\left(t_{n}-t\right)+\tau_{n} v\right)\right| \leq e^{\mu_{n}(t)} \int_{0}^{t} \delta_{n}(s) e^{-\mu_{n}(s)} d s
$$

for all $t \in\left[0, t_{n}\right]$ with the abbreviations

$$
\begin{aligned}
& \delta_{n}(s):=\operatorname{dist}\left(\left.\frac{d}{d t}\left(z_{n}\left(t_{n}-t\right)+\tau_{n} v\right)\right|_{t=s},-F\left(z_{n}\left(t_{n}-s\right)+\tau_{n} v, t_{n}-s\right)\right) \\
& \mu_{n}(s):=\int_{0}^{s} \lambda\left(t_{n}-\tau\right) d \tau=\int_{t_{n}-s}^{t_{n}} \lambda(\sigma) d \sigma .
\end{aligned}
$$

First, $\tilde{z}_{n}\left(t_{n}\right) \notin K$ results indirectly from the initial condition $\widetilde{z}_{n}(0)=\widetilde{y}_{n}+\tau_{n} \cdot v \notin \vartheta_{F}\left(t_{n}, K\right)$ because if $\tilde{z}_{n}\left(t_{n}\right)$ was element of $K$ then $\tilde{z}_{n}\left(t_{n}-\cdot\right)$ would be a solution of the differential inclusion $\xi^{\prime}(\cdot) \in F(\xi, \cdot)$ a.e. starting in $K$ and ending in $\widetilde{z}_{n}(0)=\widetilde{y}_{n}+\tau_{n} \cdot v$. Second,

$$
\begin{aligned}
& \delta_{n}(t)=\operatorname{dist}\left(-\widetilde{z}_{n}^{\prime}\left(t_{n}-t\right), \quad-F\left(z_{n}\left(t_{n}-t\right)+\tau_{n} v, t_{n}-t\right)\right) \\
& \leq \operatorname{dist}\left(-F\left(z_{n}\left(t_{n}-t\right), t_{n}-t\right),-F\left(z_{n}\left(t_{n}-t\right)+\tau_{n} v, t_{n}-t\right)\right) \\
& \leq \lambda\left(t_{n}-t\right)\left|z_{n}\left(t_{n}-t\right)-\left(z_{n}\left(t_{n}-t\right)+\tau_{n} v\right)\right|=\lambda\left(t_{n}-t\right) \cdot \tau_{n}|v|
\end{aligned}
$$

and thus for each $t \in\left[0, t_{n}\right]$,

$$
\left|\tilde{z}_{n}(t)-\left(z_{n}\left(t_{n}-t\right)+\tau_{n} v\right)\right| \leq e^{\mu_{n}(t)} \int_{0}^{t} \lambda\left(t_{n}-s\right) \tau_{n}|v| \cdot 1 d s \leq e^{\mu_{n}(t)} \mu_{n}(t) \tau_{n}|v| .
$$

So, in particular, $\widetilde{z}_{n}\left(t_{n}\right) \notin K$ has the wanted form $\widetilde{z}_{n}\left(t_{n}\right)=z_{n}(0)+\tau_{n} \cdot\left(v+w_{n}\right)$ with $z_{n}(0) \in K, 0<\tau_{n} \leq \frac{1}{n}$ and $\left|w_{n}\right| \leq|v| e^{\mu_{n}\left(t_{n}\right)} \mu_{n}\left(t_{n}\right) \longrightarrow 0$ for $n \rightarrow \infty$. 
Proof of Proposition 2.4. " $\supset$ " results immediately from the definition of Liminf since $K=\vartheta_{F}(0, K)$ holds obviously.

" $\subset$ " is now proven indirectly: Assume there exists some $w \in T_{K}^{C}\left(x_{0}\right)$ not belonging to the

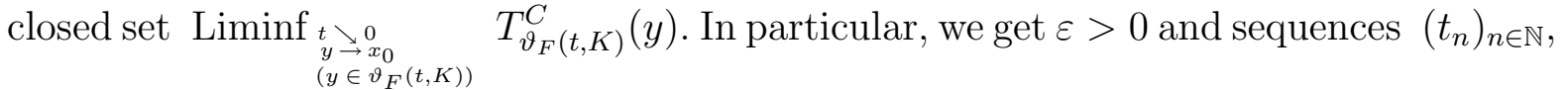
$\left(y_{n}\right)_{n \in \mathbb{N}}$ converging to 0 and $x_{0}$ respectively and satisfying $t_{n} \geq 0, y_{n} \in \vartheta_{F}\left(t_{n}, K\right)$, $w \notin \mathbb{B}_{\varepsilon}\left(T_{\vartheta_{F}\left(t_{n}, K\right)}^{C}\left(y_{n}\right)\right)$ for all $n \in \mathbb{N}$. The last property implies $\mathbb{B}_{\varepsilon}(w) \cap T_{\vartheta_{F}\left(t_{n}, K\right)}^{C}\left(y_{n}\right)=\emptyset$. Applying now Lemma 3.4 to every element $v \in \mathbb{B}_{\varepsilon}(w)$, we conclude $v \notin\left(T_{K}^{C}\left(x_{0}\right)\right)^{\circ}$, i.e. $\mathbb{B}_{\varepsilon}(w) \cap\left(T_{K}^{C}\left(x_{0}\right)\right)^{\circ}=\emptyset$. Clarke tangent cone $T_{K}^{C}\left(x_{0}\right)$, however, is always convex closed and has nonempty interior by assumption. So $T_{K}^{C}\left(x_{0}\right)$ is the closure of its interior and, we conclude that $w$ has to be in the interior of the complement of $T_{K}^{C}\left(x_{0}\right)$-contradicting the assumption $w \in T_{K}^{C}\left(x_{0}\right)$.

Proof of Theorem 2.5. Assuming that the statement is false, there must exist sequences $\left(t_{n}\right)_{n \in \mathbb{N}},\left(y_{n}\right)_{n \in \mathbb{N}}$ with $0<t_{n+1}<t_{n}<\frac{1}{n}, y_{n} \in \vartheta_{F}\left(t_{n}, K\right), T_{\vartheta_{F}\left(t_{n}, K\right)}^{C}\left(y_{n}\right)^{\circ}=\emptyset$ for each $n$. As mentioned before, the linear growth assumption about $F$ and Gronwall's Lemma ensure $\operatorname{dist}\left(y_{n}, K\right) \leq \operatorname{const}(F, K) \cdot t_{n}$ for all $n \in \mathbb{N}$. Due to the compactness of $K \subset \mathbb{R}^{N}$, there is a subsequence (again denoted by) $\left(y_{n}\right)_{n \in \mathbb{N}}$ converging to some $x_{0} \in K$ additionally. Supposing $K \subset \mathbb{R}^{N}$ to be epi-Lipschitzian, Lemma 3.1 implies $T_{K}^{C}\left(x_{0}\right)^{\circ} \neq \emptyset$.

In combination with $T_{\vartheta_{F}\left(t_{n}, K\right)}^{C}\left(y_{n}\right)^{\circ}=\emptyset$ for all $n \in \mathbb{N}$, this property will lead to a contradiction by means of Proposition 2.4: $\quad$ Each $T_{\vartheta_{F}\left(t_{n}, K\right)}^{C}\left(y_{n}\right) \subset \mathbb{R}^{N}$ is a closed convex cone with empty interior. Thus it is contained a $(N-1)$-dimensional subspace of $\mathbb{R}^{N}$ and let $p_{n} \in \mathbb{R}^{N}$ denote a unit vector orthogonal to this subspace, i.e. particularly $\left\langle p_{n}, v\right\rangle=0$ for all $v \in T_{\vartheta_{F}\left(t_{n}, K\right)}^{C}\left(y_{n}\right)$. Due to the compactness of the unit sphere in $\mathbb{R}^{N}$, there exists a subsequence $\left(p_{n_{j}}\right)_{j \in \mathbb{N}}$ converging to a unit vector $p \in \mathbb{R}^{N}$.

Applying Proposition 2.4 to $\left(y_{n_{j}}\right)_{j \in \mathbb{N}}$ and $\left(t_{n_{j}}\right)_{j \in \mathbb{N}}$, we finally obtain $\langle p, v\rangle=0$ for all $v \in T_{K}^{C}\left(x_{0}\right)$ because each $v \in T_{K}^{C}\left(x_{0}\right)$ is limit of a sequence $\left(v_{j}\right)_{j \in \mathbb{N}}$ with $v_{j} \in T_{\vartheta_{F}\left(t_{n_{j}}, K\right)}^{C}\left(y_{n_{j}}\right)$ and thus, $\left\langle p_{n_{j}}, v_{j}\right\rangle=0$ for each $j \in \mathbb{N}$.

This orthogonality $p \perp T_{K}^{C}\left(x_{0}\right)$, however, is contradicting the assumption $T_{K}^{C}\left(x_{0}\right)^{\circ} \neq \emptyset$.

\section{References}

[1] L. Ambrosio, Geometric evolution problems, distance function and viscosity solutions, in : Buttazzo, G. (ed.) et al., Calculus of variations and partial differential equations. Topics on geometrical evolution problems and degree theory, Springer, 2000

[2] J.-P. Aubin, Mutational and Morphological Analysis. Tools for Shape Evolution and Morphogenesis, Birkhäuser, 1999

[3] J.-P. Aubin and A. Cellina, Differential Inclusions, Springer, 1984

[4] J.-P. Aubin and H. Frankowska, Set-Valued Analysis, Birkhäuser, 1990

[5] J. Benoist, Approximation and regularisation of arbitrary sets in finite dimension, Set-Valued Anal. 2 (1994), No.1-2, pp.95-115 
[6] J.M. Borwein, Epi-Lipschitz-like sets in Banach space: theorems and examples, Nonlinear Anal. 11 (1987), No. 10, pp.1207-1217

[7] J.M. Borwein and H.M. Strojwas, Tangential approximations, Nonlinear Anal. 9 (1985), No.12, pp.1347-1366

[8] A. Bressan, On two conjectures by Hájek, Funkcial. Ekvac. 23 (1980), pp.221-227.

[9] P. Cannarsa and H. Frankowska, Interior sphere property of attainable sets and time optimal control problems, ESAIM Control Optim. Calc. Var. 12 (2006), pp.350-370

[10] F.H. Clarke, Optimization and Nonsmooth Analysis, Wiley-Interscience, 1983

[11] F.H. Clarke, Yu.S. Leyaev and R.J. Stern, Complements, approximations, smoothings and invariance properties, J. Convex Anal. 4 (1997), No.2, pp.189-219

[12] T. Lorenz, Generalizing mutational equations for uniqueness of some nonlocal first-order geometric evolutions, to appear in Set-Valued Analysis

[13] T. Lorenz, Boundary regularity of reachable sets of control systems, Syst.Control Lett. 54, No.9 (2005), pp.919-924

[14] R.T. Rockafellar, Clarke's tangent cones and the boundaries of closed sets in $\mathbb{R}^{n}$, Nonlinear Anal. Theor. Meth. Appl. 3 (1979), pp.145-154

[15] R.T. Rockafellar and R. Wets, Variational Analysis, Springer, 1998

[16] J.A. Sethian, Level Set Methods and Fast Marching Methods, Cambridge Univ. Press, 1999

[17] R. Vinter, Optimal Control, Birkhäuser, 2000 